\title{
MECHANISM OF DERANGEMENTS OF THE MEDIAL SEMILUNAR CARTILAGE AND THEIR MANAGEMENT*
}

\author{
Arthur J. Helfet, Cape Town, South Africa
}

\section{MECHANICS OF THE KNEE JOINT}

The rotation element-The key to the function of the knee joint, and to its derangements, is the rotation of the tibia on the femur which occurs on flexion and extension movements. The knee is not a simple hinge joint. The opening and shutting of the front of the joint in the act of flexion and extension involves the tibia in a winding course set by the configuration of the medial condyle of the femur (Fig. 1). As the tibia passes on the femur from the fully flexed to the fully extended position it descends and then slowly ascends the curves of the femoral condyle and rotates outwards. These movements are reversed as the tibia passes back on the femur to the fully flexed position. The movement of the tibial tubercle, which is easily observed, confirms this spiral action. Moreover it shows that rotation occurs throughout

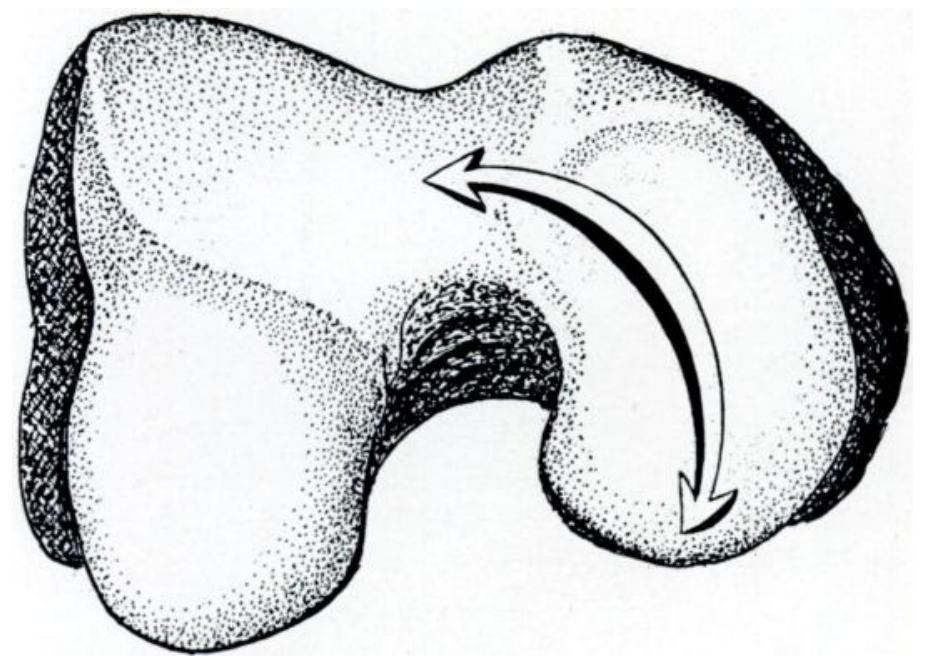

Fig. 1

The articular surface of the medial condyle of the femur showing the helicoid track followed by the tibia in flexion and extension of the knee.

the whole range of movement and not, as was thought previously, only in the last few degrees of flexion and extension. The term helicoid hinge would represent this spiral movement precisely. The extent of the rotary movement is roughly equivalent to half the width of the patella. When the knee joint is fully flexed, the tibial tubercle is in line with the medial half of the patella; in the extended knee it is in line with the lateral half of the patella (Figs. 2 and 3).

The anatomy of the muscles and ligaments of the knee facilitates this pattern of movement. The quadriceps group of muscles, running from without inwards, rotate the tibia outwards as the knee straightens. During flexion the popliteus anchors the femur, while the medial hamstrings rotate the tibia inwards. It should be noted that extension and lateral rotation of the tibia are synchronous, as are flexion and medial rotation, both being controlled by the thigh muscles. It is my belief that forcible prevention of this synchronous movement is most often responsible for internal derangement of the knee.

* The substance of this paper was presented in a Hunterian Lecture delivered at the Royal College of Surgeons of England on June 17, 1958. 
Only when the thigh muscles are relaxed is it possible to rotate the tibia on the femur freely in both directions. Even in relaxation it is not possible to achieve full extension or full flexion without full lateral or medial rotation as the case may be. As the tibia moves

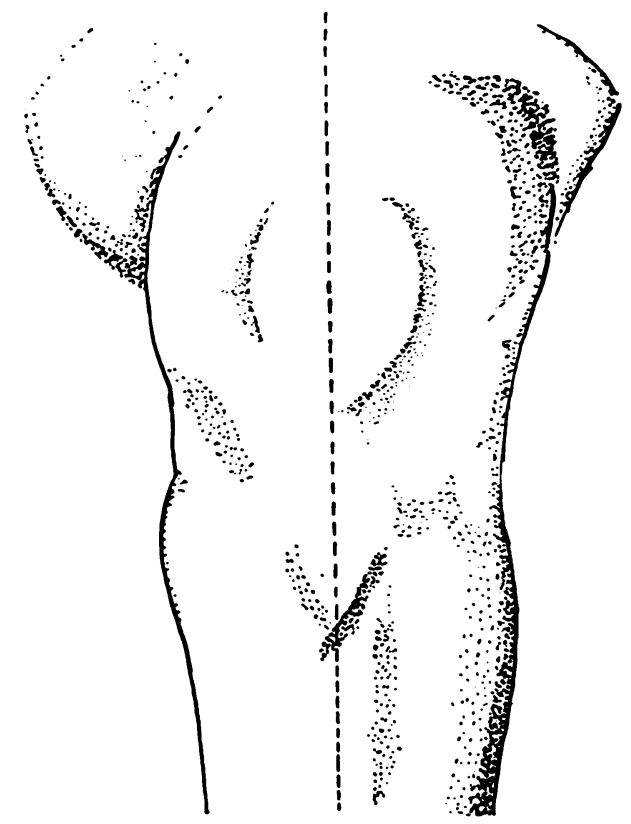

Fig. 2

Figure 2-With the knee flexed to a right angle the tibial tubercle points to the midline of the patella. Figure 3-In the fully extended knee the line of the tibial tubercle is near the lateral border of the patella.

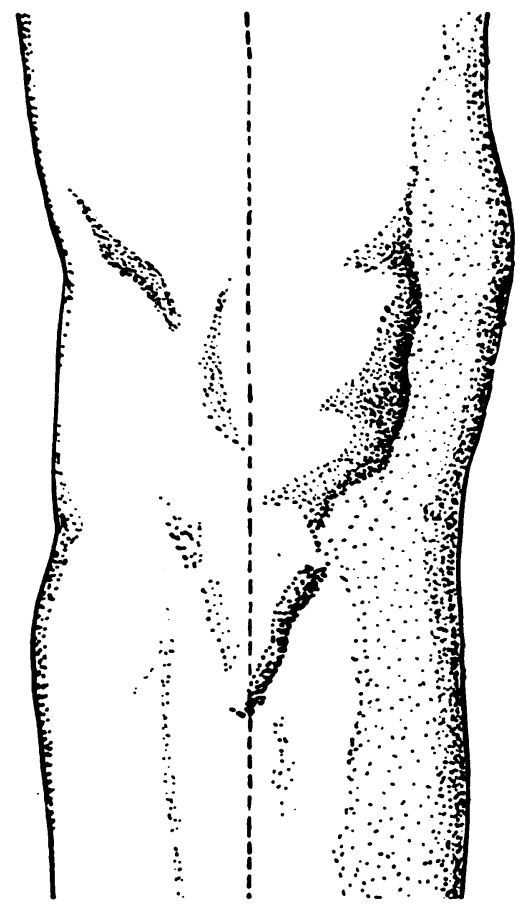

Fig. 3

from full extension to flexion its full range of free rotation increases. It should be emphasised, however, that when the quadriceps and hamstrings are active, only synchronous movement in each direction is normally possible: when the knee straightens the tibia must rotate laterally and as it flexes the tibia must rotate medially.

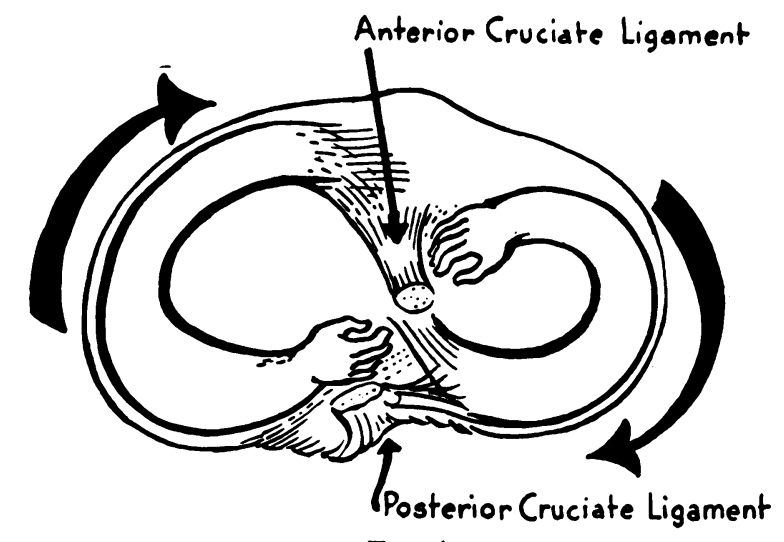

Fig. 4

Diagrammatic representation of the guiding action of the cruciate ligaments and semilunar cartilages in the rotator mechanism of the knee joint.

In a previous paper (Helfet 1948) I drew attention to the fact that the cruciate ligaments act not as stays to prevent antero-posterior displacement of the femur on the tibia, but as guide ropes to keep the tibia on its winding path when the knee extends and flexes. Further 
consideration suggests that the semilunar cartilages join the cruciate ligaments in this function in the manner suggested diagrammatically in Figure 4, where the semilunar cartilages represent an extension of the cruciate ligaments in this function of guiding the rotation of the tibia on the femur. It is probable that both the cruciate ligaments and the semilunar cartilages are of similar origin, for the cartilages are differentiated from the the same embryological layer in continuity with the cruciate ligaments and are not truly cartilaginous. It seems likely that they function as an accessory part of the rotator mechanism (Kaplan 1955).

The continuity of the ligamentous and cartilaginous structures, in what Sir Harry Platt aptly termed "figures-of-eight" anatomy, was accurately described by Galeazzi in 1927. Three of his illustrations, reproduced here, emphasise this characteristic. Figure 5 shows the strong fibrous band, sometimes a centimetre wide, connecting the posterior horn of the lateral cartilage to the femoral attachment of the anterior cruciate liagment. Figure 6 shows the fibrous bands which connect the anterior cruciate ligament with the anterior horn of the medial and with the anterior horn of the lateral cartilage. Figure 7 shows Barkow's ligament connecting the posterior horn of the lateral cartilage and the anterior horn of the medial cartilage.

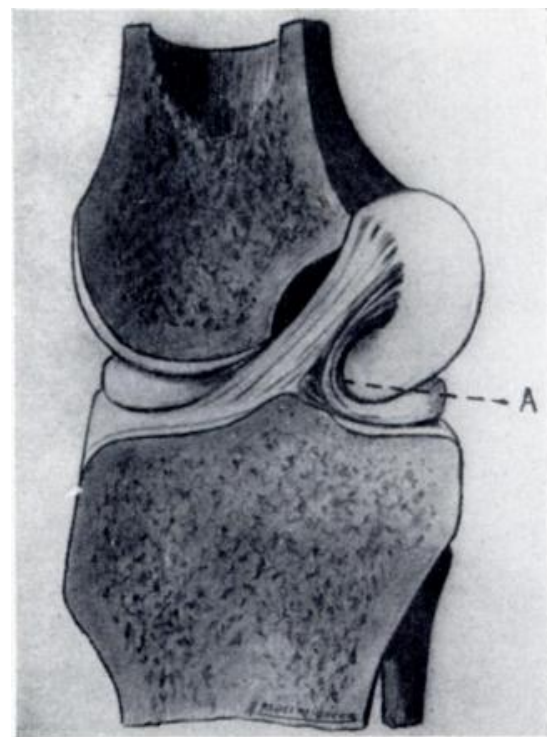

Fig. 5

Strong fibrous band, sometimes a centimetre wide, connecting the posterior horn of the lateral cartilage to the femoral attachment of the anterior cruciate ligament. (From Galeazzi 1927, by permission of the Editor of the Journal of Bone and Joint Surgery.)

Galeazzi also referred to the transverse ligament of the knee (Winslow), which joins the anterior horns of the two cartilages, and emphasised particularly the firm attachments between the anterior cruciate ligament and medial cartilage. All this is important to my thesis.

Watson-Jones (1955) drew attention to the part played by the semilunar cartilages in the rotator mechanism when he described what might be called the retreating cartilage. "Place one finger over the joint line of the knee in front of the medial ligament where the

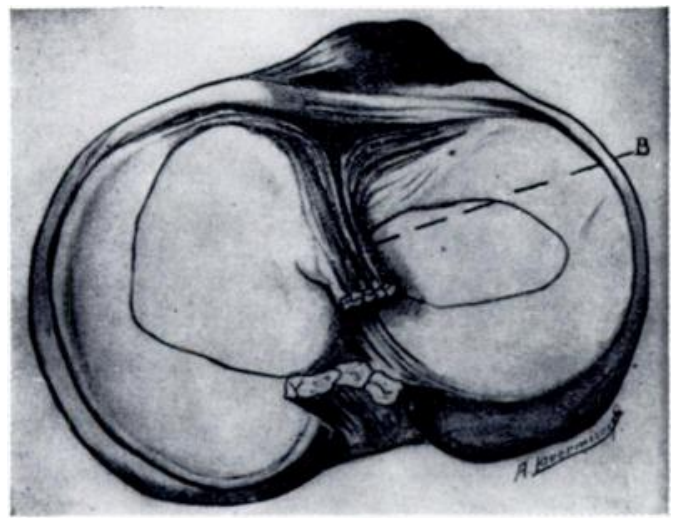

FIG. 6

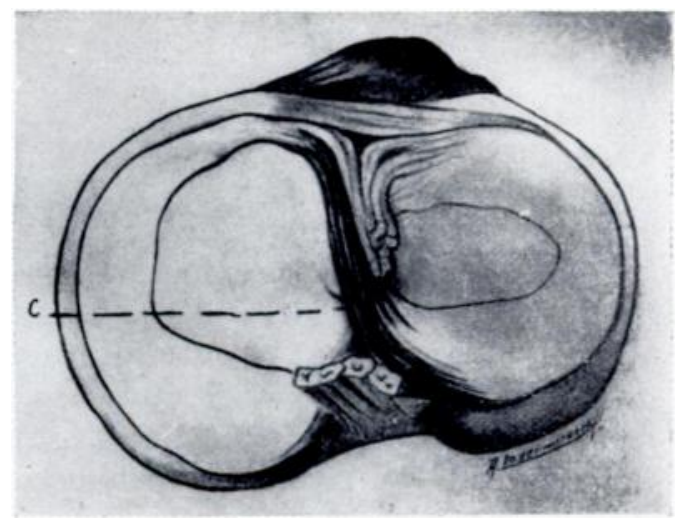

Fig. 7

Figure 6-Fibrous bands which connect the anterior cruciate ligament with the anterior horn of the medial, and B, with the anterior horn of the lateral cartilage. (From Galeazzi 1927, by permission of the Editor of the Journal of Bone and Joint Surgery.) Figure 7-Barkow's ligament connecting posterior horn of lateral cartilage to anterior horn of medial cartilage. (From Galeazzi 1927, by permission of the Editor of the Journal of Bone and Joint Surgery.)

VOL. 41 B, NO. 2, MAY 1959 
curved margin of the medial femoral condyle approaches the tibial tuberosity. Now rotate the foot and leg laterally. It is easy to feel the medial semilunar cartilage disappearing from the surface, leaving a sulcus between the bones." This sign is present when the knee is flexed and the muscles relaxed and is presumably an expression of the independence of the figure-ofeight mechanism, of which the cartilage is only a part, when rotation is unaccompanied by synchronous extension of the knee. Normal lateral rotation of the tibia when the knee is straightened does not produce this sign: the cartilage maintains its relationship to the margin of the tibia.

On the other hand, when, as will be shown, rupture of the cartilage blocks lateral rotation of the tibia, absence of the "retreating" cartilage sign in the flexed knee may be used diagnostically.

The patella-The patella is a pulley, and its excursion is controlled by the direction of action of the quadriceps group of muscles and the position of the tibial tubercle which carries the patellar ligament.

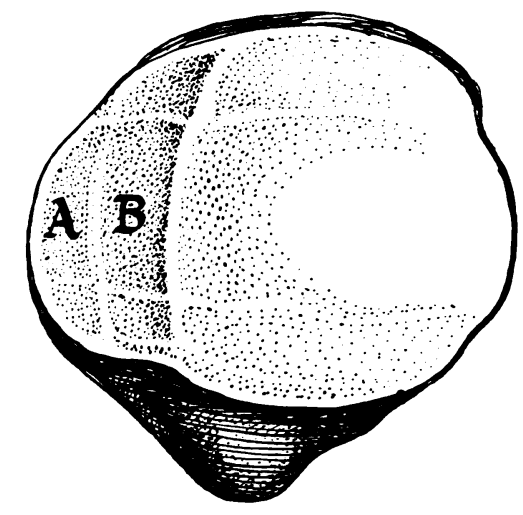

FIG. 8

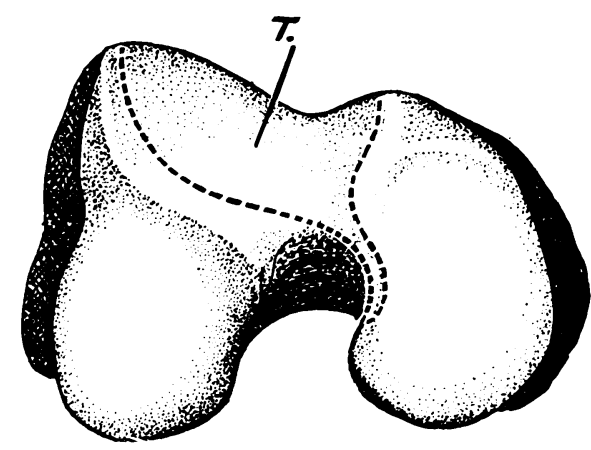

FIG. 9

Figure 8-Patella showing smaller medial slope with vertical division into two planes $A$ and $B$. Figure 9-Lower end of right femur showing trochlear surface (T), for patella, extending along inner condyle as an area with which patella articulates in flexion. (From Frazer's Anatomy of the Human Skeleton, by permission of Messrs J. \& A. Churchill.)

The articular surface of the patella is divided into a large lateral and a smaller medial surface. These surfaces are separated by a vertical rounded ridge (Fig. 8). In full extension its shape fits comfortably and evenly into the trochlear surface of the femur. The ridge then lies in the hollow or trough of the trochlear surface. When the knee is bent the patella is carried downwards and backwards on the under aspect of the femur where the trochlear surface is prolonged on to the inner condyle (Fig. 9). In flexion the patella tilts away from the lateral condyle, so that only the inner part of its articular surface rests against the medial condyle (Frazer 1958).

So long as the tibial tubercle rotates smoothly, the patella travels its short course smoothly and under even tension. However, any derangement of the joint which prevents lateral rotation of the tibia during extension of the knee would affect normal tension, because contraction of the quadriceps would force the inner border of the patella against the medial condyle of the femur. This explains the patellar symptoms and signs produced by certain cartilage injuriessuch as retropatellar pain on climbing and descending stairs, tenderness of the medial border of the patella, and the pattern of cartilage erosion which develops on the medial surface only of the patella and femur. This pattern differs from that produced by retropatellar arthritis complicating recurring dislocation, in which case the lateral surface of the patellar cartilage undergoes fibrillation (Langston 1958). 


\section{MECHANISM OF INTERNAL DERANGEMENTS OF THE KNEE}

Types of violence-With weight bearing, the knee joint straightens and the tibia rotates laterally. It rotates medially when the knee flexes. If this synchrony is forcibly prevented-as by the weight of the falling body - the rotator mechanism of the knee is injured. Certain cartilage tears are caused by nothing more than this disruption of the rotator mechanism of the knee.

Figure 10 shows a common manner of injury of the footballer's knee. He catches his toe, trips and falls while the foot and tibia are outwardly rotated: flexion occurs without medial rotation.

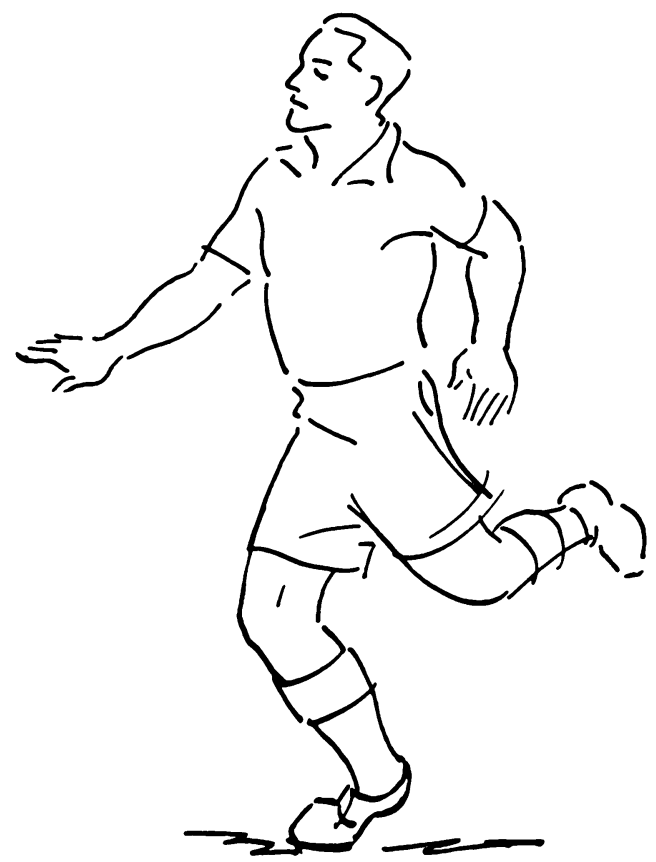

Fig. 10

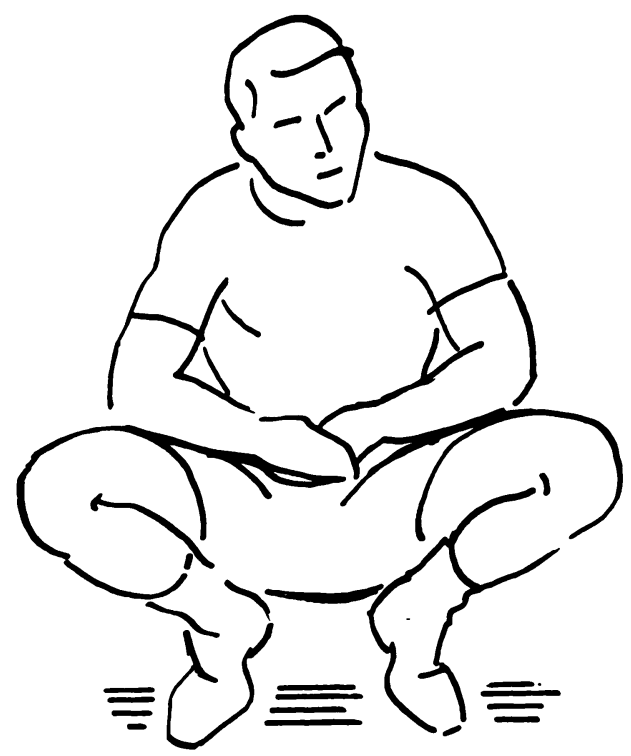

Fig. 11

Figure 10-The footballer catches his toe, trips and falls. The foot and tibia are outwardly rotated while the knee flexes. Figure 11 -An uncontrolled twist while weight is taken on the fully flexed knee may tear the medial cartilage.

Figure 11 shows the position in which miners and those who do housework frequently injure the medial cartilage. An uncontrolled twist-rotation without synchronous extensionis followed by a searing pain, the signal of a tear in the cartilage. One patient, a carpenter, was working in front of a chest of drawers in this attitude. A drawer had jammed. He wrenched vigorously to open it. The drawer gave suddenly. He twisted on his right knee and immediately he felt a searing pain over the medial cartilage. To relieve the agony he threw himself on to his left knee, with identical consequences to that joint. At operation buckethandle tears of both medial cartilages were found.

In another instance a woman knelt on her kitchen sink to open a cupboard above it. The door opened suddenly and she twisted on the flexed knee. A stab of pain signalled detachment of the anterior end of her medial cartilage.

\section{MEDIAL CARTILAGE LESIONS}

It seems that if synchronous rotation is prevented, flexion is achieved either by pulling the medial cartilage away from its anterior attachments (Figs. 14 and 16) or by splitting the cartilage longitudinally to allow the free border to bowstring across the joint (Figs. 13 and 15).

VOL. $41 \mathrm{~B}$, NO. 2, MAY 1959 


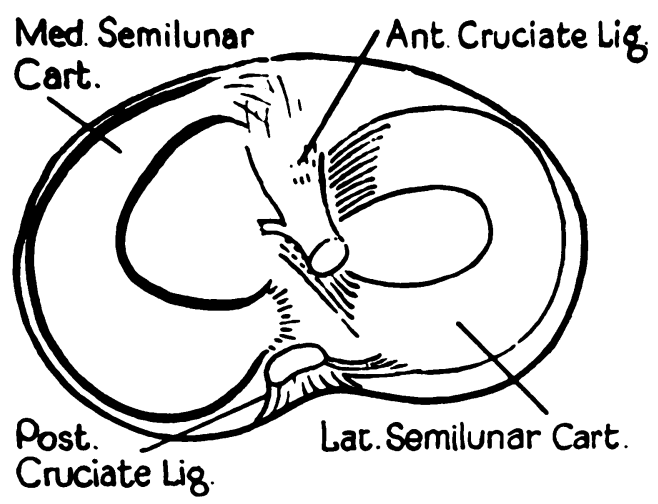

Fig. 12

The relationship of the semilunar cartilages and cruciate ligaments.

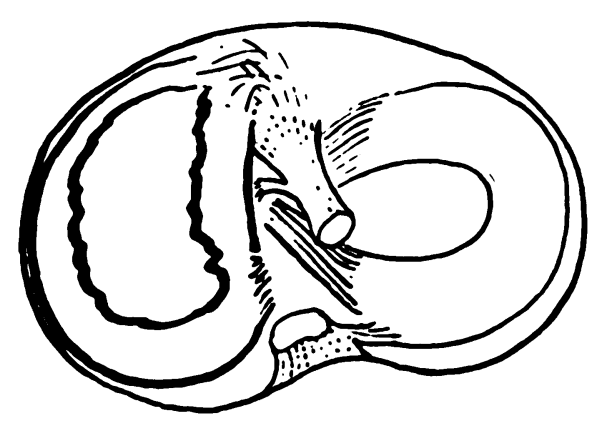

FIG. 13

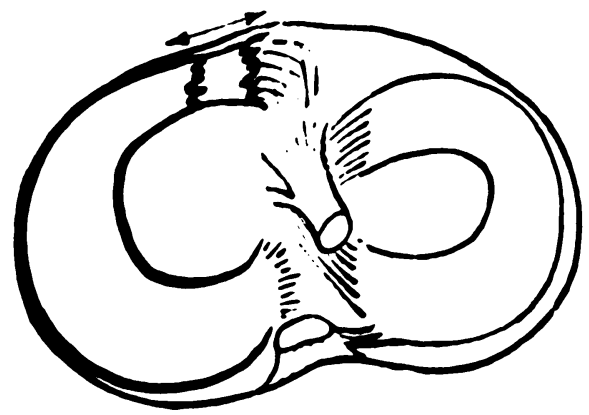

FIG. 14

Figure 13-The bucket-handle or bowstring tear of the medial semilunar cartilage. The separation is roughly equivalent to the distance the tibia normally rotates. Figure 14-The retracted cartilage: rupture of the anterior horn of the medial cartilage from its ligamentous attachments.

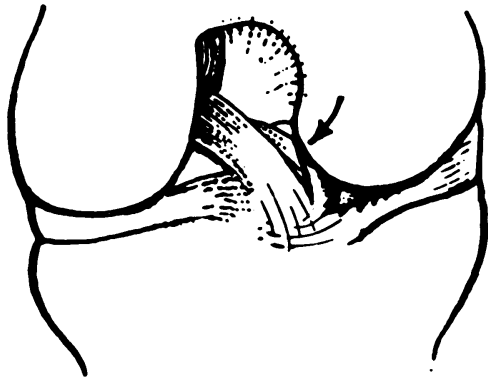

Fig. 15

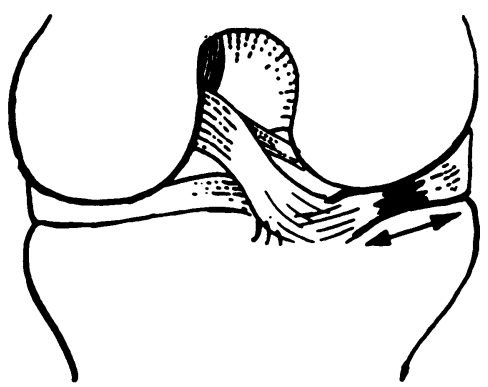

Fig. 16

Figure 15-Diagrammatic presentation of the bowstring cartilage as seen from the front. Figure 16-Diagrammatic presentation of the retracted cartilage as seen from the front. 
As the complete range of rotation is at most half the width of the patella the displacement need not be extensive, and it is always within this measurement. In either case the figure-ofeight rotator mechanism is interrupted. As the anterior end of the cartilage is detached the whole cartilage retracts and a gap forms between the attachment of the cruciate ligament and the torn edge. At operation the gap may be recognised and, in a young patient, the free edge of the cartilage will present a ruffle which raises it from the surface of the tibia. The front half of the cartilage is slack and looks too long: by the inexperienced it may be considered a "lax cartilage." In an older patient, with fibrotic and less resilient cartilages, retraction causes a thickening of the anterior half of the cartilage, which is yellowish, hard and triangular in section and a heaped-up mass is formed in the anterior compartment of the joint. The lump so formed produces a pattern of erosion over the corresponding area of articular cartilage on the antero-medial part of the weight-bearing surface of the medial femoral condyle (Fig. 17). As this type of cartilage tear presents its own clinical features and sequelae, I submit that it

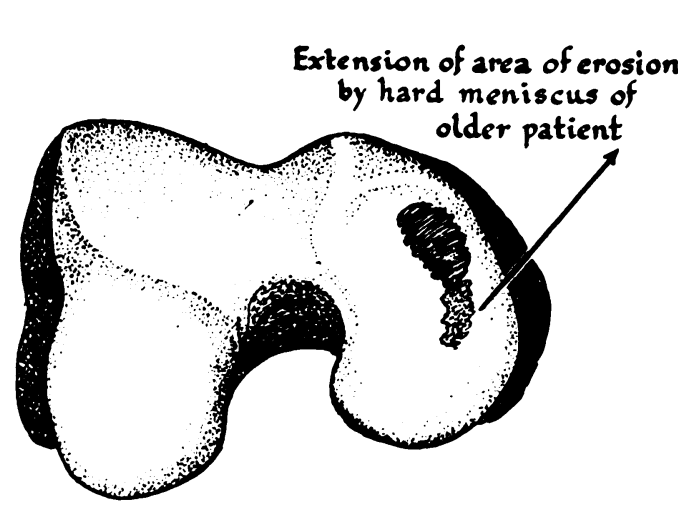

Fig. 17

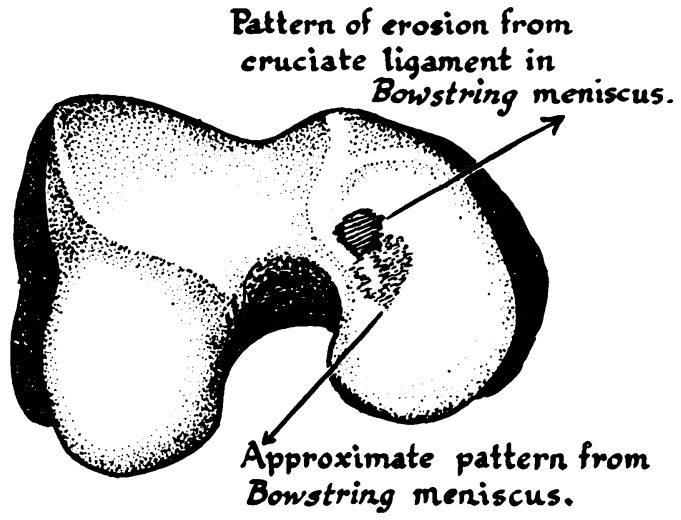

Fig. 18

Figure 17-Pattern of erosion caused by heaped-up retracted cartilage. Figure 18-Pattern of erosion caused by bowstring cartilage.

should be known as the "retracted" cartilage to distinguish it from the "bucket-handle" cartilage, which itself should more aptly be named the "bowstring" cartilage. In the latter type of injury the whole, or part, of the cartilage is bowstrung across the joint, and the symptoms are more severe than in the case of the retracted cartilage. But if at the first attack or subsequently the bowstring snaps and coils up at the fixed end in the anterior compartment, the clinical picture resembles that of the retracted variety. The " bowstring" injury produces a pattern of erosion on the articular cartilage covering the intercondylar border of the condyle of the femur (Fig. 18) (Helfet 1948).

In November 16, 1883, Thomas Annandale (1885) performed the first recorded operation for displaced semilunar cartilage. He found " that the internal semilunar cartilage was completely separated from its anterior attachment to the tibia and was displaced backwards about half an inch." He did not excise the cartilage but cured his patient by drawing the cartilage forward and stitching it to its former attachment. Ten weeks later the man was back at his work with a normally functioning knee. This historical note not only records the " retracted" cartilage for the first time, but may well indicate an approach to treatment.

Less frequently the posterior end of the cartilage is torn or detached. Only once have I found the whole back end of the cartilage wrenched from its attachments and the entire cartilage coiled in the anterior compartment. This injury was suffered by an amateur in a wrestling match, but we were unable to reconstruct the exact mechanism. 
A review of a recent personal series of 232 operations for cartilage injuries reveals that retracted and bowstring cartilages are the most frequent consequences of injury ( 80.6 per cent) (Table I). In the whole series the bowstring tears exceeded the retracted cartilages by 11 per cent, but with increasing age the relative incidence of anterior tears increased (Table II). This must mean that as the cartilage becomes less resilient and more fibrotic, it is more liable to tear from its ligamentous moorings than to split in its substance.

TABLE I

Types of Cartilage InJury (232 Cases)

\begin{tabular}{lllllrrr|}
\hline & & & & \multicolumn{2}{c}{ Number } & Per cent \\
\hline Retracted cartilage &. &. &. &. & 81 & $34 \cdot 9$ \\
"Bowstring" or bucket-handle cartilage & 106 & $45 \cdot 7$ \\
Posterior tears &. &. &. &. &. & 26 & $11 \cdot 2$ \\
Other tears &. &. &. &. &. & 19 & $8 \cdot 2$ \\
\hline
\end{tabular}

TABLE II

Type of Injury Related to Age of Patient

\begin{tabular}{|llcccc|}
\hline Age $($ years $)$ & $\cdot$ & $10-19$ & $20-44$ & 45 and over \\
\hline Retracted cartilage.$\cdot$ & $14(35 \%)$ & $53(43 \%)$ & $14(58 \%)$ \\
“Bowstring” cartilage & $26(65 \%)$ & $70(57 \%)$ & $10(42 \%)$ \\
\hline
\end{tabular}

\section{CLINICAL FEATURES}

Restriction of movement-Tears and displacements of the medial semilunar cartilage affect the smooth action of the rotator mechanism of the knee, and the presence of injury is signified in every instance by inability to rotate the tibia laterally to its full extent and, therefore, to extend the knee completely.

The bowstring cartilage blocks these movements. To call it "locking" of the joint is inaccurate because only extension and lateral rotation are prevented. Flexion and medial rotation are unaffected and give comfort to the injured joint. The retracted cartilage has the same effect but is not so painful. At the start I reasoned that, since the anterior cruciate ligament exerted its guiding action on the tibia in part through the medial cartilage, anterior detachment of the cartilage would weaken this function and leave the tibia unable to complete its normal excursion. But this cannot be so, for after excision of the torn cartilage the quadriceps regains full control of the range of rotation and incidentally of extension of the tibia. The cartilage is an accessory factor in the rotator mechanism. The abnormal cartilage disturbs function, but when it is excised the knee can compensate for its absence.

It appears that any increase in volume of the contents of the antero-medial compartment of the knee prevents full lateral rotation of the tibia. This can be demonstrated experimentally at operation. Through the usual medial incision with the knee bent over the end of the table, the lateral rotation of the tibia during extension shows very well. If one puts a sterile rubber catheter across the medial joint space, the tibia cannot rotate fully. The reaction to the anterior tear is therefore a blocking of movement by the "heaping-up" of the retracted cartilage. 
This blocking of movement of rotation and therefore of extension gives us the following four clinical signs which are pathognomonic of displacement or swelling of the medial cartilage. 1) Forced passive extension produces finger-point pain over the site of injury in the antero-medial compartment of the joint. The patient can locate the pain exactly. 2) Any attempt to force the final degree of lateral rotation will cause pain localised to the same spot. Flexion and medial rotation of the knee are not affected and, indeed, give the patient comfort. This statement needs a word of qualification, because if the effusion in the knee is tense, flexion is limited and painful because of the distension of the capsule as a whole; and if the split in the bowstring cartilage extends to the posterior compartment the final degrees

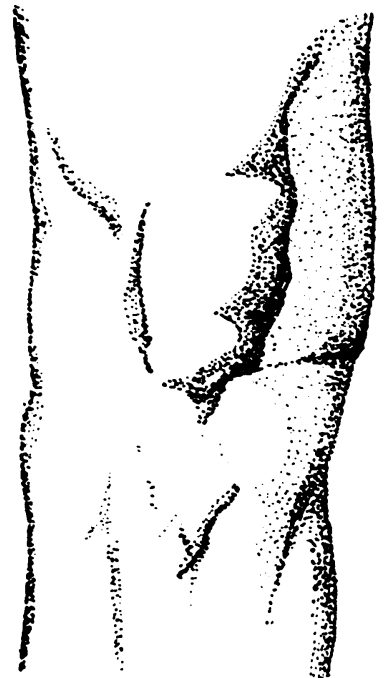

FIG. 19

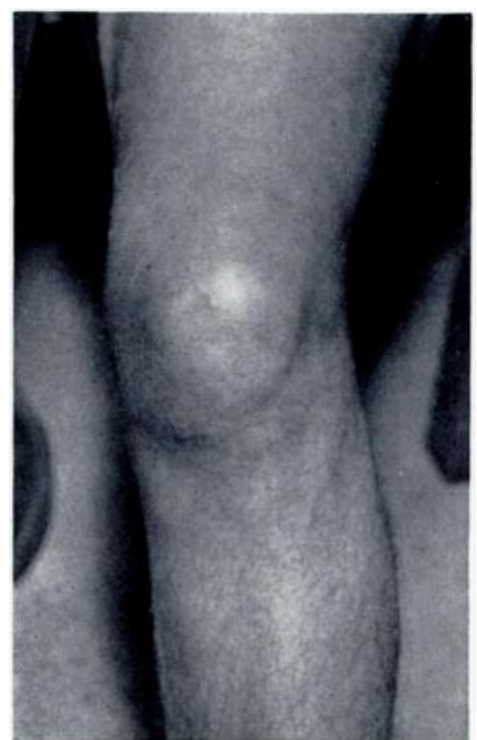

Fig. 20

Figure 19-Diagram of the " blocked " knee showing: 1) that line of tibial tubercle remains near the midline of the patella; and 2) that the medial femoral condyle protrudes over the margin of the tibia. Figure 20-The protrusion of the medial femoral condyle and the blocked rotation of the tibial tubercle are obvious in this knee, with a retracted medial cartilage.

of flexion and medial rotation may also be obstructed. 3) Compared with the normal knee the outward excursion of the tibial tubercle is limited. When the normal knee is straightened the tibial tubercle reaches the line of the lateral border of the patella. The tibial tubercle of the injured knee reaches approximately to the central axis of the patella (Figs. 19 and 20). 4) The final screw-home of the knee in completing extension aligns the borders of the medial femoral condyle and the adjacent tibia exactly. When the screw-home is prevented by an injured cartilage, the femoral condyle is left protruding over the margin of the tibia. Unless the knee is very swollen this is obvious to the eye (Fig. 20). In any case, it can be detected by palpation. If the examining finger follows the subcutaneous surface of the tibia upwards it will, when it reaches the knee, be obstructed by the protruding medial condyle of the femur, a feature which may be confirmed by comparison with the normal knee. The protrusion is most obvious in the knee in which arthritic changes have become established.

At operation for a torn medial cartilage these signs may be confirmed. After opening the joint through a medial incision, straighten the knee. The medial femoral condyle overlaps the tibial margin. Remove the cartilage and straighten the knee again. Complete extension is obtained and the femur and tibia are in perfect rotational alignment.

VOL. 41 B, NO. 2, MAY 1959 
Tenderness-After the injury the whole cartilage may be sensitive. but after a while the retracted cartilage will be tender in the anterior compartment. and the cartilage with a posterior tear in the posterior compartment. The complete bowstring cartilage may be tender at both ends. In the case of an untreated tear tenderness subsequently develops in two other areas. The retracted cartilage, by virtue of its local increase in bulk in the anterior compartment, gradually erodes an area of weight-bearing articular cartilage on the medial femoral condyle (Fig. 17). The spot is adjacent to the femoral condyle protrusion (previous paragraph) and is most easily located when the knee is flexed. The other site of tenderness is the medial border of the patella and the adjacent medial slope of the trochlear surface of the femur; it is the consequence of injury to articular cartilage by undue tension between these surfaces of the patella and femur.

\section{PAIN AND ITS MECHANISM}

It is interesting to establish a pathological basis for the various sites and types of pain that follow injury to the medial cartilage. The bowstring tear causes immediate and severe pain. The patient cannot continue to take weight on the leg. During the next two or three weeks the symptoms improve, though forcing the knee straight or turning suddenly remain painful movements. With time the cruciate ligaments stretch, the patient develops slight anteroposterior laxity, and the knee becomes more comfortable. Each recurrence of the derangement produces less severe symptoms until finally the articular cartilage is eroded and the symptoms of " arthritis " develop-namely, aching and swelling after effort, subsiding with rest, and pain and aching referable mainly to the medial compartment of the knee. Later, when the patella becomes involved, the disconcerting symptom is increased pain on descending and ascending stairs, from increased pressure between the medial border of the patella and the medial trochlear surface of the femur.

The injury that causes the "retracted" cartilage gives less pain. The patient may experience a sharp momentary pain, which is relieved when the twist is corrected. The joint swells, and there are recurrent stabs of pain on sudden movements. Later, when the articular cartilage is eroded, the more distressing symptoms of arthritis become manifest. These are pain in the medial compartment of the knee on walking, and aching after effort and later at night. The patient may feel that he is " walking with a stone in the knee." The ache is localised to the same area. The patellar symptoms, when they develop, are like those produced by the bowstring cartilage.

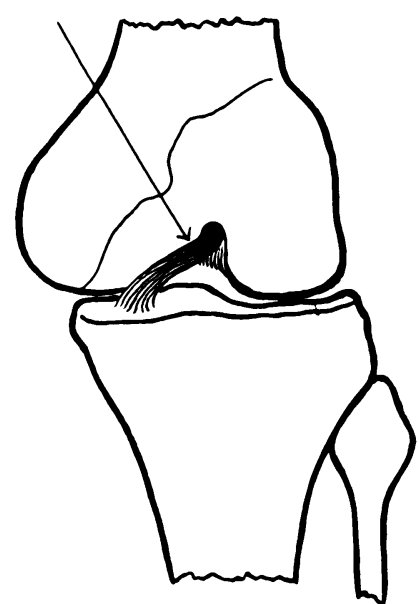

FiG. 21
ARTICULAR EROSIONS

The essence of normal function of the knee is synchrony of movement and weight bearing without undue tension at any time. In a previous article (Helfet 1948) the suggestion that the cruciate ligaments acted as guide ropes, and not as straps to check movement, carried this implication. The tension in the cruciate ligaments in all normal - and therefore synchronousmovements remains even. If the ligaments acted as checks

Fig. 21

The arrow indicates the point at which the anterior cruciate ligament hooks over the edge of the medial condyle of the femur if rotation is prevented during extension. Recurrent or longstanding "locking" of the knee causes erosion of the articular cartilage there and inflammation of the corresponding part of the cruciate ligament. Note the loss of rotational alignment between the femur and tibia. (Reproduced from the Lancet by permission of the Editor.) 
to antero-posterior movement then at times the tension would increase. In normal movements this does not happen. The experiments on cadaveric knees (Galeazzi 1927) to demonstrate increase in tension are basically unsound because in each case movement is tested in only one direction, and any movement without its synchronous counterpart is an abnormal movement.

Conversely, when rotation is prevented during extension, the cruciate ligament is tensed. It impinges on the lateral edge of the medial femoral condyle and in time erodes the surface of the articular cartilage in this area. In turn the cruciate ligament suffers from the continuous or recurrent tension and is slowly stretched, with consequent laxity of the joint; at operation it may show a raw area of granulation on its otherwise shining surface (Fig. 21).

The effect of the retracted cartilage differs with age. The youthful soft cartilage is heaped up or ruffled. The antero-medial part of the weight-bearing surface of the medial femoral condyle comes into contact with the thickened anterior part of the retracted cartilage when the

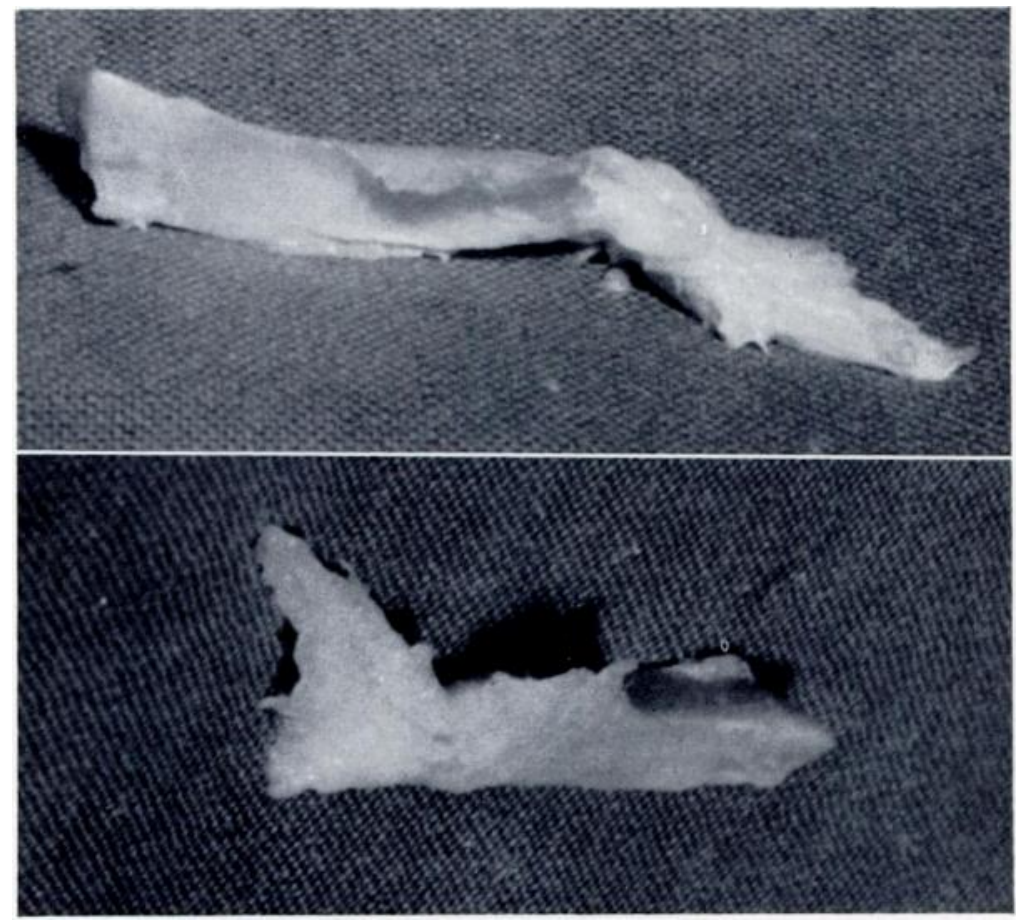

FIG. 22

Two views showing the thickened triangular shape of the front part of the retracted cartilage and the splayed squashed edge at the site of fracture.

knee is extended, and is slowly worn away (Fig. 17). But, because of the protrusion of the femoral condyle, the area of erosion never reaches the edge. Consequently, after the torn cartilage is removed and full extension and rotational alignment of femur and tibia are regained, normal cartilage is brought back to the weight-bearing area: this is one of the reasons why it is worth while removing the retracted cartilage, however long its history and whatever the age of the patient.

At operation upon older patients the cartilage may be seen to be yellowish and hard. It forms a shorter triangle on section because the free edge has contracted. The narrow hard cartilage tends to erode a more linear groove on the femoral condyle and the area of erosion seems to extend farther backwards (Fig. 17). At this age, too, transverse tears in the middle third of the cartilage are more common (Fig. 22). The torn back end of the front part has a splayed, squashed and scalloped edge. It seems that the heaping up or thickening caused by 
the anterior retraction is all taken up in this front portion and that eventually the weightbearing surfaces nip it off at this junction between thickened and normal portions of the cartilage.

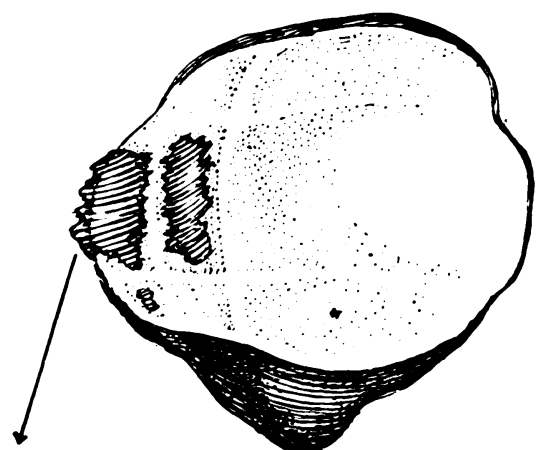

Proliferative heaping up of Cartilage.

FIG. 23

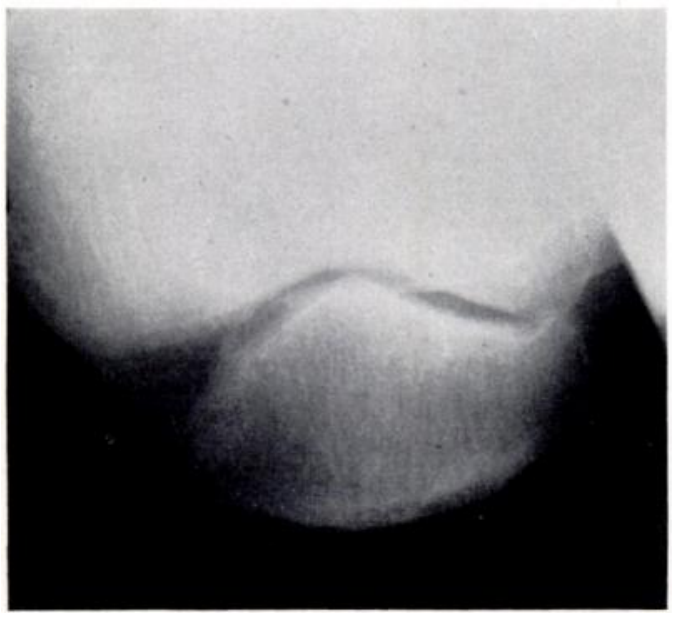

Fig. 24
FIG. 23

Maximum erosion over areas $\mathbf{A}$ and $\mathbf{B}$ of Figure 8 with proliferative changes along the medial border of the patella.

Figure 24-Axial view of right patella showing erosion of articular cartilage in area B and of corresponding area of trochlear surface of femur. Figure 25-Degenerative changes on medial articular surface of patella.

FiG. 26

Pattern of erosion of medial trochlear surface of femur from patella.

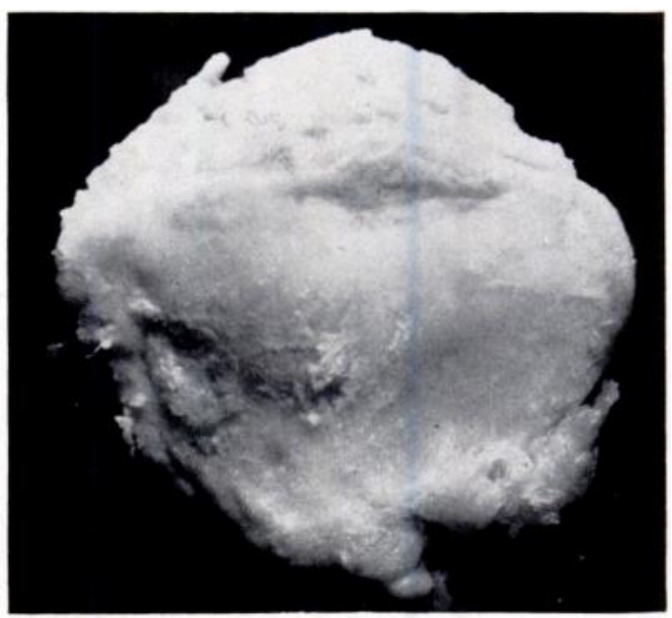

FIG. 25

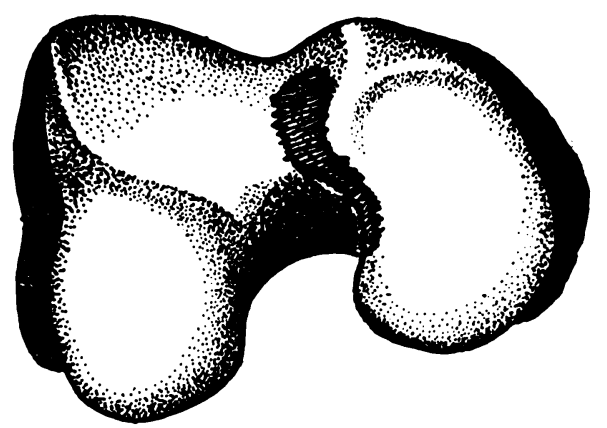

FIG. 26

The third pattern of erosion concerns the medial border of the patella and the surface of the femur with which it is in contact. Attention has been drawn to the effect produced by inadequate excursion of the tibial tubercle on the relationships of the patella and femur. 
Normally, in full extension, the line of action of the quadriceps on the patellar ligament settles the patella comfortably on the trochlear surface of the femur. When the tibial tubercle halts short of this line the patella will deviate medially as the knee extends, and particularly when the quadriceps contracts against increased resistance as in climbing and descending stairs. It is tensed against the medial slope of the trochlear surface of the femur and the tension is greatest over the area A and B in Figure 8 of the patella and area T of Figure 9 of the femur. These are the sites of articular cartilage erosion. (See also Figures 23 to 26.)

During flexion from complete extension the increased medial pull on the whole mechanism produces increased pressure by the patella as it slips downwards and backwards on the extension of the trochlear surface on the medial condyle of the femur, and gradually the complete pattern of traumatic arthritis of the knee is etched.

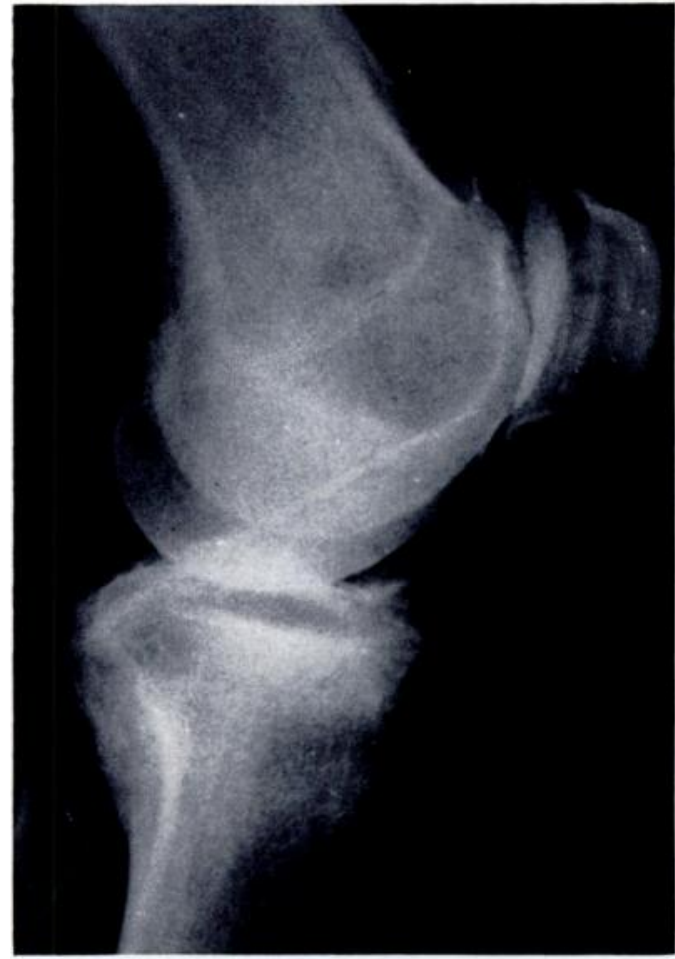

FIG. 27

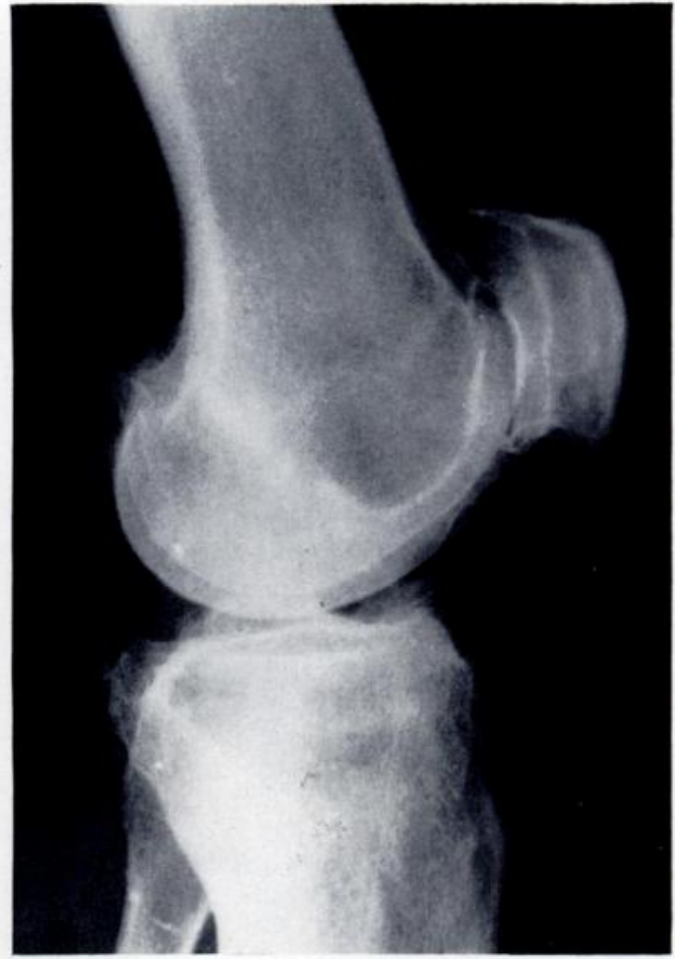

Fig. 28

Case 1. Figure 27-The preoperative radiograph shows that in the limited extension possible the patella is squeezed against the undersurface of the medial femoral condyle and there is erosion of joint surface between the patella and trochlear surface of the femur. Figure 28-Comparable radiograph of the knee after removal of the medial cartilage. The knee is straighter and the patella tips back into the trochlear space.

Normally, in the flexed position the patella tilts on to its medial articular surface. This tilt is accentuated in the blocked knee and will be present even in the position of fullest extension.

\section{CASE REPORTS}

Case 1-Figures 27 and 28 show lateral radiographs of the knee of a man of seventy-two with a longstanding retracted medial cartilage. The radiograph taken before operation shows that in the limited extension possible the patella is squeezed against the undersurface of the medial femoral condyle and there is erosion of the joint surface between the patella and the trochlear surface of the femur. In the radiograph taken after removal of the medial cartilage the knee is seen to be straighter and the patella tips back into the trochlear space.

This patient had no trouble with the knee until January 1957, when he twisted it. Since the accident he had been unable to straighten the knee completely and walking had been painful. These 


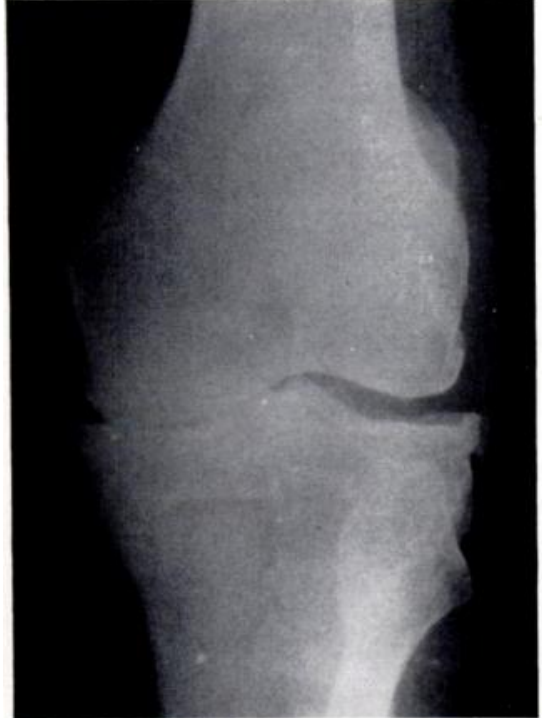

Fig. 29

Case 1-Showing so-called arthritis of the knee. symptoms gradually worsened, and he suffered a gnawing ache in bed at night. When walking he felt as if there was a stone or lump in the medial compartment of the knee: this was relieved by bending the knee, and he tended to limp with the knee in a more and more flexed position.

On examination, extension was limited by fully 20 degrees. The anterior horn of the medial cartilage and the articular surface of the femoral condyle were acutely tender. The signs of femoral protrusion and lack of rotation of the tibia were positive (Fig. 29). Radiographs showed erosion of the femoral articular surface and of the patella, with osteophyte formation.

At operation the medial compartment of the knee was opened. When the knee was extended the medial condyle of the femur protruded and lateral rotation of tibia was restricted. After removal of the medial cartilage rotation of the tibia was immediately restored and the knee could be extended fully. There were areas of erosion on the medial femoral condyle and on the medial facet of the patella. The findings are illustrated in Figures 30 to 36.

Progress after operation was rapid. The knee could be extended almost completely. The gnawing ache disappeared, and three months after the operation he was able to walk and climb stairs without pain.

Comment-I have deliberately presented the history of a patient who had extensive arthritis with erosion due to a torn medial cartilage, for it shows that even at a late stage the simple excision of a cartilage is desirable. In the earlier stage not only are symptoms relieved and

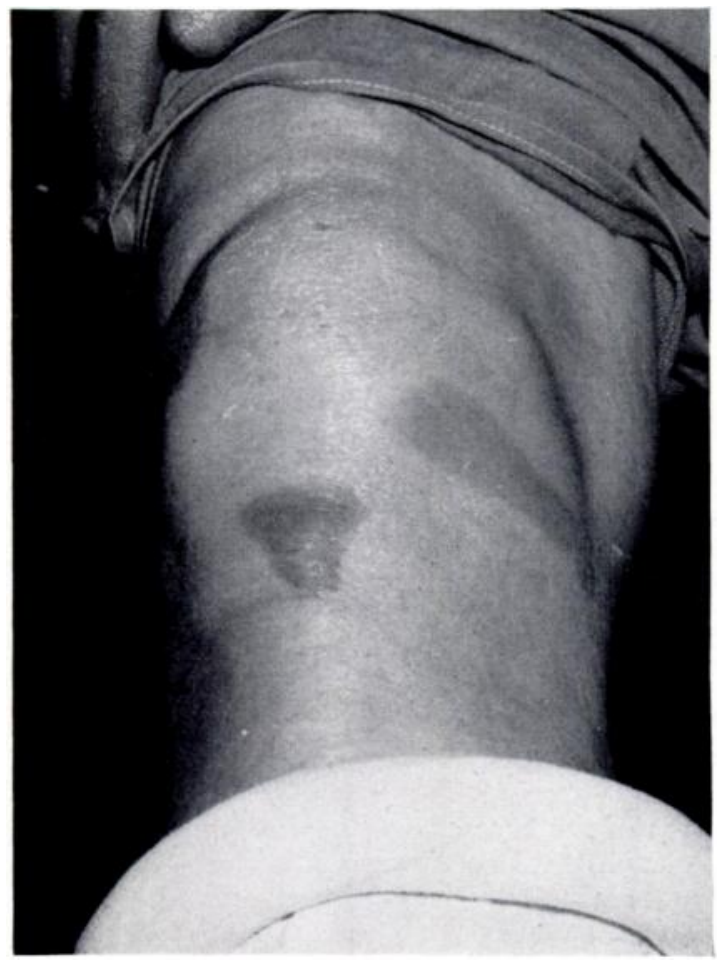

FIG. 30

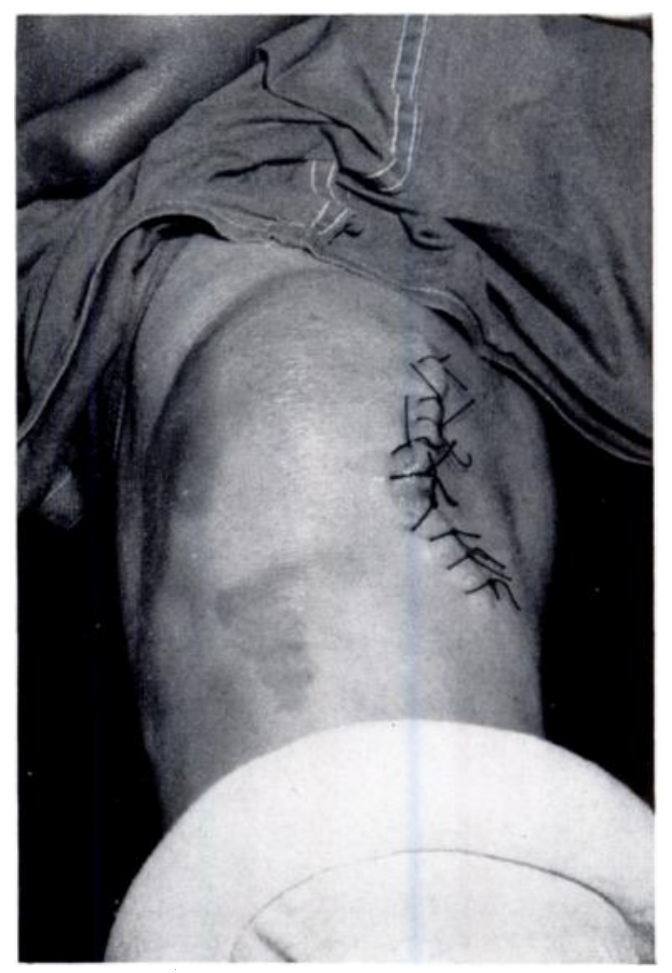

Fig. 31

Case 1. Fig. 30-Shows knee extended, with protrusion of medial condyle of femur and limited lateral rotation of tibial tubercle. Figure 31-After removal of medial cartilage. Immediately the tibia rotates the knee extends fully. 


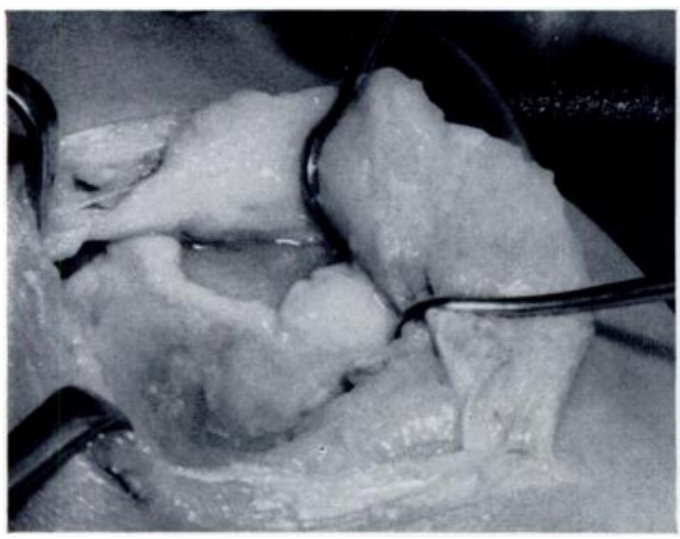

FIG. 32

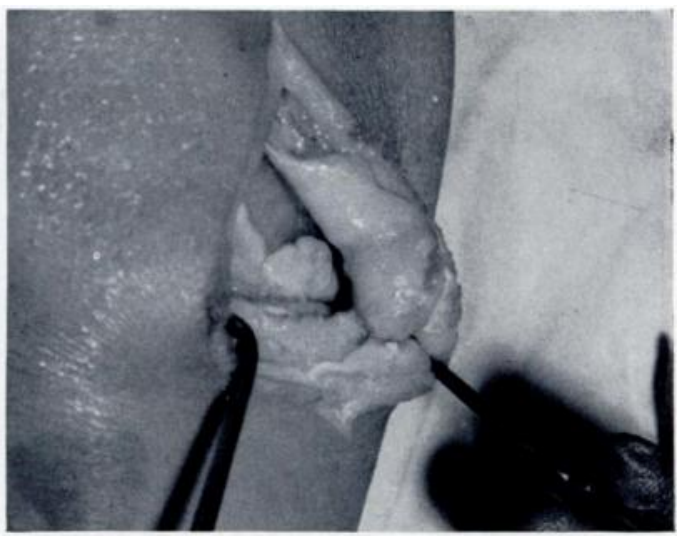

Fig. 33

Case 1. Figure 32-Knee extended. Protrusion of femoral condyle over margin of tibia. Lower hook grips retracted cartilage. Note presence and heaping up of articular cartilage on presenting surface of femur. Area of erosion is in relation to the cartilage. Figure 33-Knee extended. After removal of cartilage. Realignment of margins of femoral condyle and tibia.

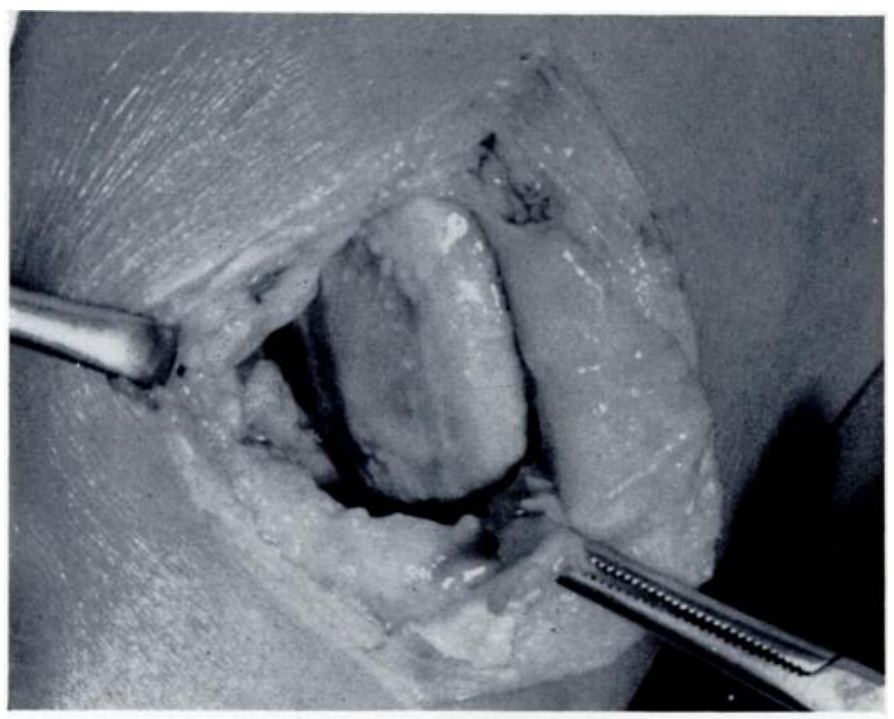

FIG. 34

Case 1-Compare with Figure 17.

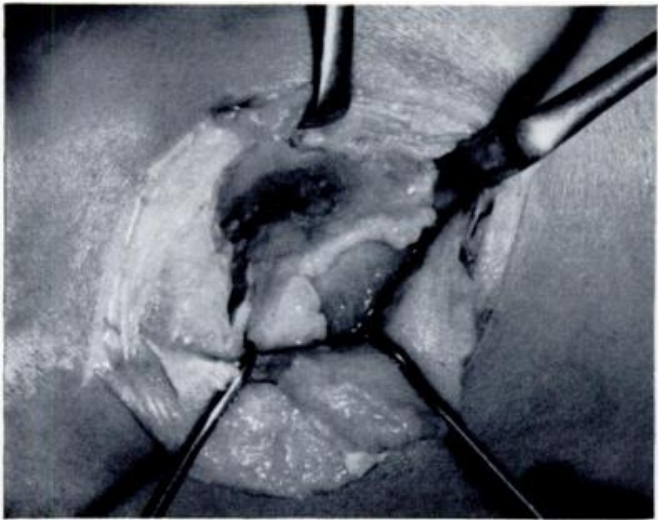

Fig. 35

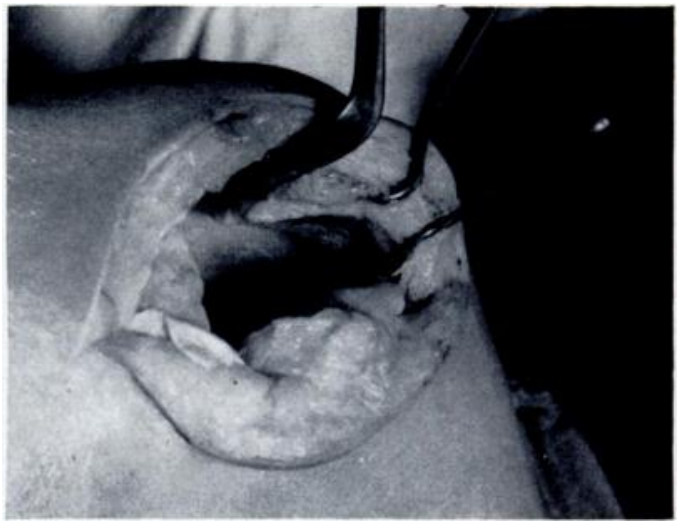

FIG. 36

Case 1. Figure 35-Knee extended. Patella retracted. Area of erosion on medial slope of trochlear surface. Figure 36-Showing the smooth unaffected lateral slope of the trochlear surface of the femur.

VOL. 41 B, NO. 2, MAY 1959 


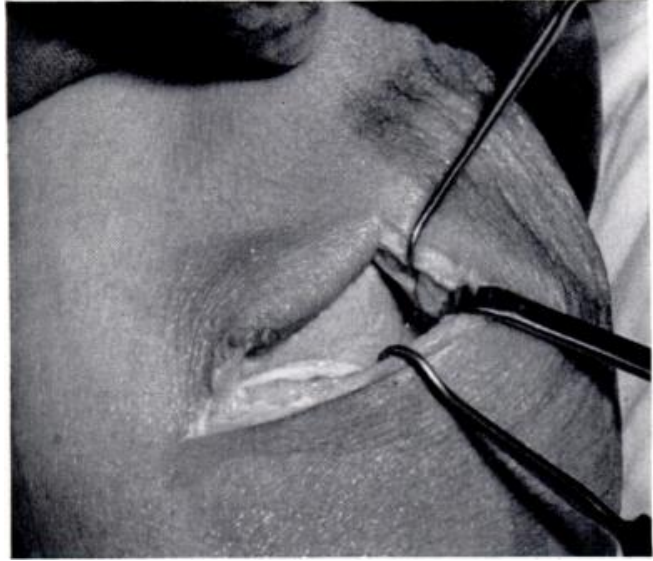

FIG. 37

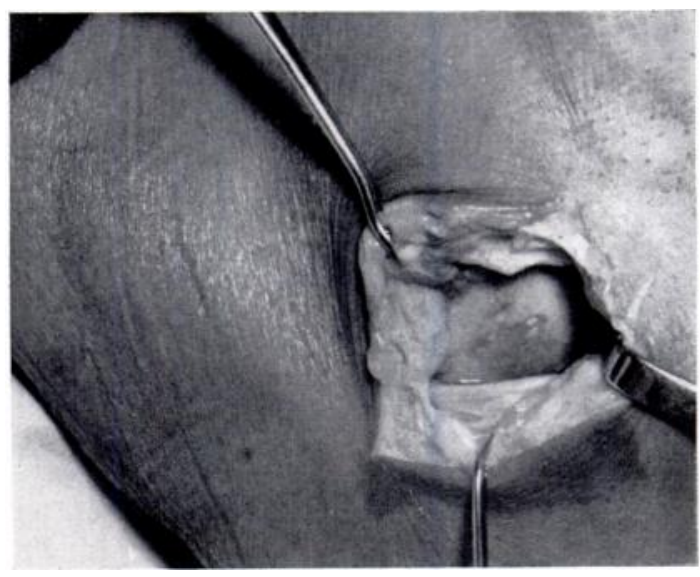

FIG. 38

Case 2. Figure 37-Shows the linear erosion on the anterior part of the articular surface of the femoral condyle. Figure 38-Shows the erosion on the femoral condyle, linear in the anterior part and broadening out farther back.

function restored, but further degeneration is prevented. After removal of the cartilage the patient is taught to extend the knee fully. Normal articular cartilage is brought into the weight-bearing area and, once more, the contracting quadriceps allows the patella to travel

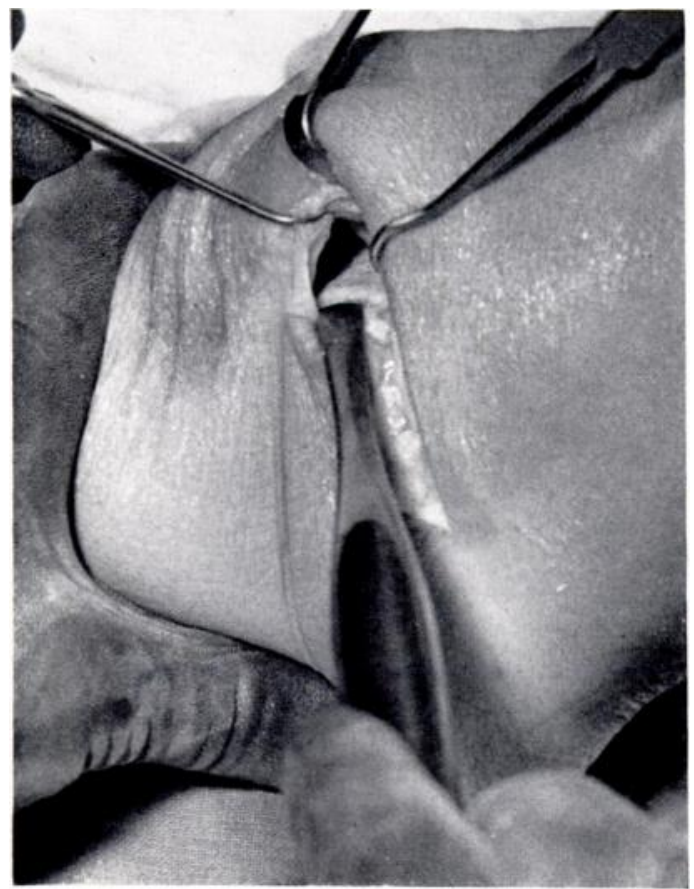

FIG. 39

Case 2-Shows the erosion of the medial slope of the trochlear surface of the femur. in the correspondingly proportioned groove of the trochlear surface of the femur. There is no tension and no pain.

Post-operative care should be planned to teach the patient once more to extend the knee completely. In older patients, especially, this is the main purpose of quadriceps retraining. Unless the patella shows gross degeneration it need not be excised. but the osteophytic protrusions on the medial border should be removed. Older patients are happier with than without the patella.

Case 2-Figures 37 and 38 illustrate the case of a man aged sixty-six. In spite of a gunshot wound of the femur during the first world war, he had enjoyed normal function and played much golf. Six months before consulting me he had fallen on the left knee. The knee was swollen and painful for three weeks. Thereafter he could again play golf but felt uncomfortable after a round, and in the last two months his knee had become progressively worse. He felt pain when he swung against the knee, and towards the end of the game his knee would ache and the leg feel lame. At first the symptoms settled down with rest but finally the pain became constant.

On examination, the four signs that I have described were all present. The knee was tender over the articular surface of the femoral condyle and chiefly on the medial side of the trochlear surface and the corresponding border of the patella. Figure 37 shows a linear erosion on the anterior part of the articular surface of the femoral condyle; the erosion broadened out farther back (Fig. 38). Figure 39 shows the erosion of the medial slope of the trochlear surface of the femur. The erosion on the patella corresponded exactly. The lateral slopes of the articular cartilage of the femur and the patella were unmarked. 
After the operation the knee straightened out completely, his convalescence was normal and full function was recovered.

Case 3-Figure 40 shows the findings at operation in a woman aged fifty-one. She did not recall any injury, but complained of intermittent pain and swelling in the right knee for three months. On examination rotation of the tibia was restricted and the femoral condyle protruded. There was tenderness over the anterior end of the medial cartilage, and along the medial border of the patella.

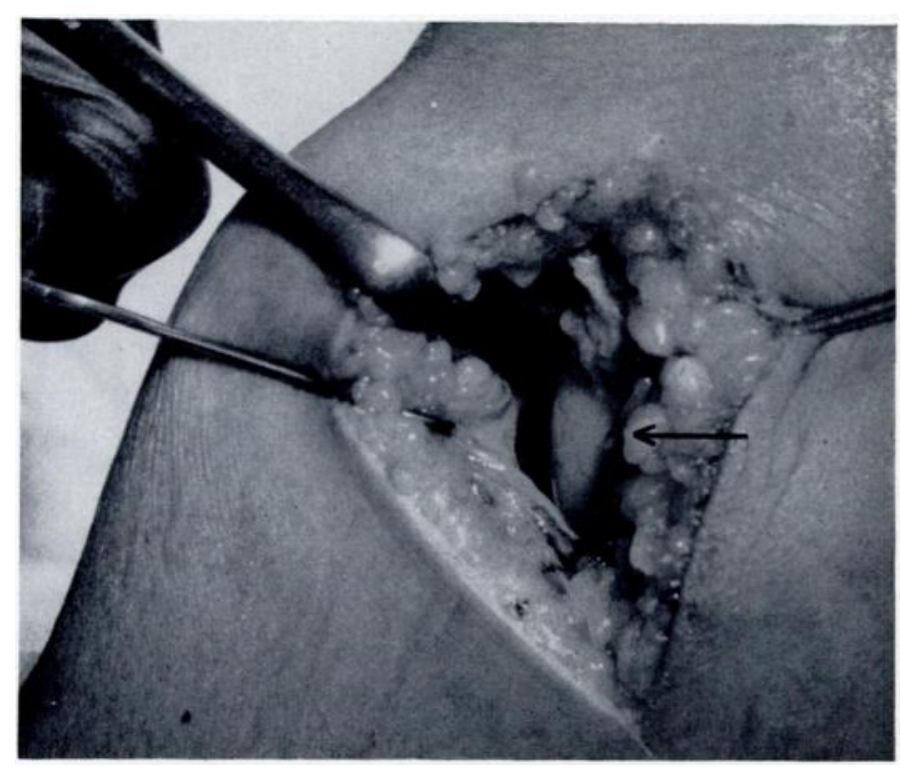

Fig. 40

Case 3-Shows the detached anterior end of the cartilage and the early erosion of the articular cartilage on the opposing femoral condyle.

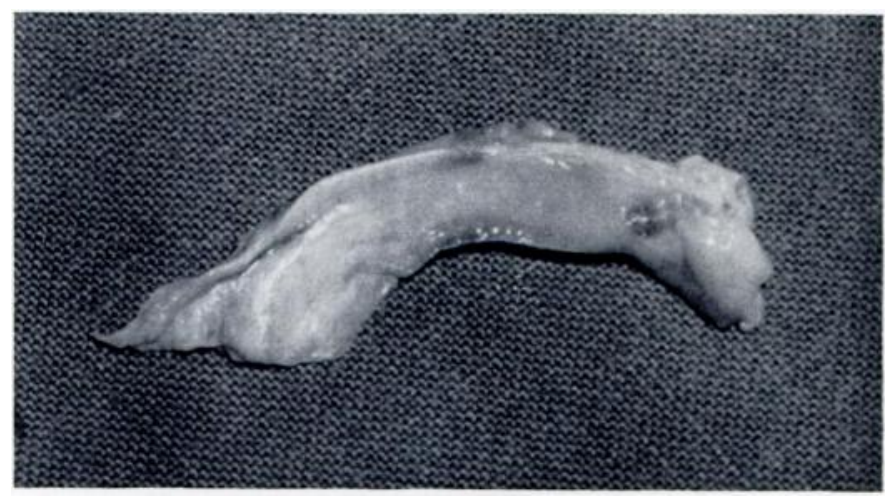

Fig. 41

Case 3-Retracted cartilage from a younger patient than that shown in Figure 22, but showing the early contraction of the front of the cartilage and the scalloped squashed edge of the fracture.

Forced passive extension caused localised pain over the anterior end of the cartilage, and forced active extension caused pain behind the patella.

Conservative treatment did not help her, and six weeks later the cartilage was removed. The cartilage was detached in front, the anterior two-thirds were thickened and there was an almost complete transverse fracture at the junction of the anterior two-thirds and posterior third. The fractured edge showed the typical scalloped squashed appearance (Fig. 41).

Six weeks later, when she had recovered good function of the quadriceps, she had a full range of comfortable movement.

VOL. 41 B, NO. 2, MAY 1959 


\section{THE LATERAL SEMILUNAR CARTILAGE}

So far I have considered only the torn medial cartilage. Most anterior tears of the lateral cartilage present the same physical signs of limited outward rotation of the tibia, but with the pain and tenderness referred to the lateral compartment. Tears of the lateral cartilage are less frequent - twenty-nine to 203 in a series of 232 cartilage injuries-and have a greater inclination to cause " giving way" of the knee. In some instances tears of the lateral cartilage and of the posterior end of the medial cartilage are characterised not by limitation of extension and lateral rotation, but by limitation of flexion and medial rotation of the tibia, pain if these movements are forced, and tenderness over the posterior compartments of the knee. To determine the exact relationships of symptoms to site of injury requires further investigation, as do the patterns of erosion on the lateral condyle of the femur. I propose to present this aspect of the subject for publication later.

\section{SUMMARY}

1. The semilunar cartilages are part of the rotator mechanism of the knee joint.

2. Movement of the weight-bearing knee comprises synchronous lateral rotation of the tibia with extension and medial rotation of the tibia with flexion.

3. When this synchrony is disturbed, injuries to the semilunar cartilages result.

4. Damage to the anterior two-thirds of the medial cartilage blocks lateral rotation of the tibia, with consequent physical signs that are pathognomonic of the retracted and the bowstring cartilage, which are the most common types of injury.

5. Each type of cartilage injury produces its own pattern of erosion of articular cartilage and its own sequence of symptoms as so-called arthritis develops.

6. The sequence of symptoms may be halted and often reversed by removal of the torn cartilage. Operation is warranted in most cases however long the history and whatever the age of the patient.

7. The development of medial retropatellar arthritis is explained. The symptoms are often relieved by removal of the medial semilunar cartilage and adequate post-operative rehabilitation.

I am indebted to Mr Bruce Franck of Cape Town for the drawings and to Mr B. Todt of Groote Schuur Hospital for the photographs.

\section{REFERENCES}

Annandale, T. (1885): An Operation for Displaced Semilunar Cartilage. British Medical Journal, i, 799. Frazer, J. E. (1958): Anatomy of the Human Skeleton. Fifth edition. Edited by A. S. Breathnach. London: J. \& A. Churchill Ltd.

Galeazzi, R. (1927): Clinical and Experimental Study of Lesions of the Semilunar Cartilages of the Knee Joint. Journal of Bone and Joint Surgery, 9, 515.

Helfet, A. J. (1948): Function of the Cruciate Ligaments of the Knee-joint. Lancet, i, 665.

Kaplan, E. B. (1955): The Embryology of the Menisci of the Knee Joint. Bulletin of the Hospital for Joint Diseases, 16, 111.

Langston, H. H. (1958): Dislocation of Patella and its Relation to Chondromalacia Patellae. British Medical Journal, i, 155.

Platt, Sir H. Personal Communication.

Watson-Jones, Sir R. (1955): Fractures and Joint Injuries. Fourth edition, vol. 2, p. 764 . Edinburgh and London: E. \& S. Livingstone Ltd. 